Gazi University
Journal of Science
http://dergipark.gov.tr/gujs

\title{
On Complex Interval Arithmetic Using Polar Form
}

\author{
Edrees M. Nori MAHMOOD ${ }^{1}$, Gültekin SOYLU ${ }^{2, *(0)}$ \\ ${ }^{1}$ University of Mosul, Department of Operations Research and Intelligent Techniques, Mosul, Iraq \\ ${ }^{2}$ Akdeniz. University, Faculty of Science, 07058 Antalya, Turkey

\section{Highlights} \\ - Presentation of sources of difficulties of addition and subtraction of sectors. \\ - Introduction of effective and low-cost algorithms solving the problem. \\ - Case studies demonstrating the superiority of the proposed algorithms.
}

\begin{tabular}{|c|}
\hline \\
\hline $\begin{array}{l}\text { Article Info } \\
\text { Received:30 Nov } 2020 \\
\text { Accepted:02 Mar } 2021\end{array}$ \\
\hline 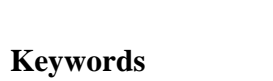 \\
\hline $\begin{array}{l}\text { Interval arithmetic } \\
\text { Complex sets } \\
\text { Polar form } \\
\text { Global optimization. }\end{array}$ \\
\hline
\end{tabular}

\section{INTRODUCTION}

In complex interval arithmetic three different types of intervals are introduced: rectangular [1-3], circular [4], and sector representation [5]. All these representations suffer from not being closed under one or more of the basic operations addition, subtraction, multiplication and division.

Whenever an operation fails to be closed for a given representation, the issue is to obtain the smallest possible rectangle, circle or sector respectively that just includes the result.

Representations based on sectors fail to be closed under addition and subtraction. Klatte and Ulrich [5] first introduced arithmetic operations on sectors. They proposed sector arithmetic as an alternative to rectangular and circular arithmetic in the complex interval space. They defined six different alternatives; all of them are based on mapping sectors to rectangles or circles, performing the operations on the chosen domain, and then returning to the original representation. Obviously, this forth and back transformations between rectangular and polar forms introduces a good deal of pessimism and is in no way optimal. Then, Flores [6] proposed algorithms for performing basic arithmetic operations on sectors; he called complex fans arithmetic. Even though all algorithms in [6] are proven mathematically and verified empirically, they require a significant amount of computation and moreover, they fail in the case where the origin is a boundary point of the sum of two sectors. Finally, Candau et al. in [7] derived analytical algorithms for the computation of the smallest sector circumscribing the sum of two sectors. Unfortunately, we realized that the algorithm 3. MinAngle proposed in [7] may not work correctly in some scenarios and we demonstrated this by presenting some counter-examples. These facts motivated this work: the research for an effective algorithm to perform addition and subtraction. 
Since the basic operations multiplication and division of sectors are defined flawlessly using real interval arithmetic our focus is addition and subtraction. For polar complex interval addition (subtraction) we will introduce a highly efficient and low cost algorithm compared to the algorithms in [6] and [7]. Sectors, from a different perspective, are two dimensional intervals on the real or complex plane. Therefore all results obtained in this work can be used not only for complex intervals but also for 2-D real intervals.

The rest of the paper is organized as follows: Section 2 presents the definition and arithmetic of polar intervals. The proposed algorithm is introduced in Section 3. In Section 3 we preferred to analyze the problem and summarize the results in an algorithm at the end rather than delivering the material in theoremproof form. Section 4 contains the implementation of the proposed algorithms and the comparison with the available algorithms in the literature. Finally, Section 5 concludes the work.

\section{POLAR COMPLEX INTERVALS}

Hereafter $\mathbb{I}(\mathbb{R})$ denotes the set of closed real intervals. Similarly, $\mathbb{I}\left(\mathbb{R}^{+}\right)$will denote the set of nonnegative real intervals.

Definition 1 Let $[\rho]=\left[\rho^{-}, \rho^{+}\right] \in \mathbb{I}\left(\mathbb{R}^{+}\right)$and $[\varphi]=\left[\varphi^{-}, \varphi^{+}\right] \in \mathbb{I}(\mathbb{R})$; the set defined by $S=\left\{s \in \mathbb{C}: s=\rho e^{i \varphi}, \rho \in[\rho], \varphi \in[\varphi]\right\}$ is called a polar complex interval (or sector), with magnitude interval $[\rho]$ and angle interval $[\varphi]$ denoted by $[\rho] e^{i[\varphi]}$.

For simplicity, and without loss of generality, we will restrict our study to the case in which $[\varphi]=\left[\varphi^{-}, \varphi^{+}\right] \in \mathbb{I}\left(\mathbb{R}^{+}\right)$, since all other cases can be reduced to the study of this one.

\subsection{Classification of Sectors According to Angle Intervals}

It is important to distinguish between types of sectors according to their angle intervals $[\varphi]$. It turns out to be sufficient to distinguish according to whether $\varphi^{+} \leq 2 \pi$ or $\varphi^{+}>2 \pi$.

- The set $S(\mathbb{C})$ is defined as the set of sectors with angle interval $[\varphi]$ satisfying $\varphi^{+} \leq 2 \pi$. Thus, for all $[\rho] e^{i[\varphi]}$ in $S(\mathbb{C})$, we have $\varphi^{+}-\varphi^{-} \leq 2 \pi, 0 \leq \varphi^{-}<2 \pi$ and $0<\varphi^{+} \leq 2 \pi$ (see Figure 1).

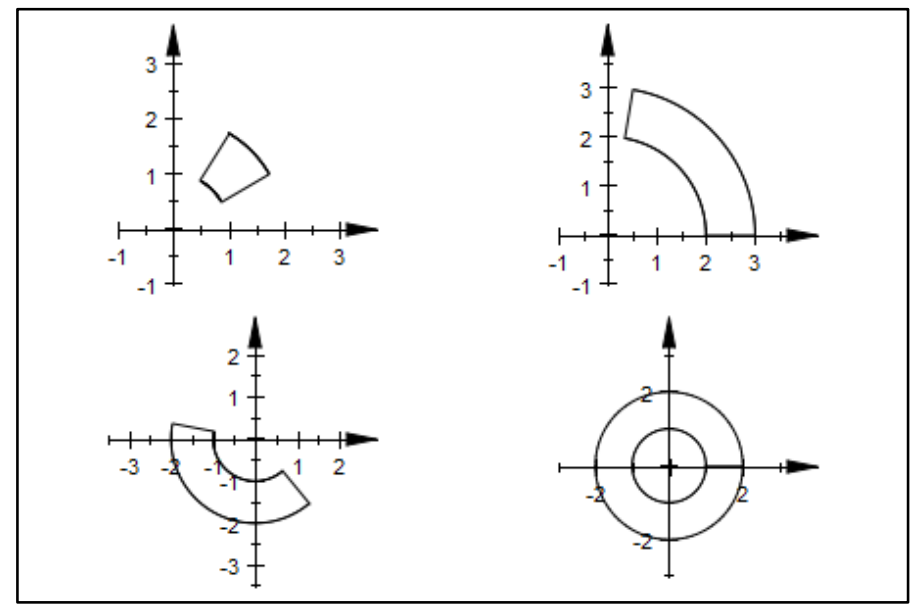

Figure 1. Some members of the set $S(\mathbb{C})$

- The set $S^{*}(\mathbb{C})$ is defined as the set of sectors with angle interval $[\varphi]$ satisfying $\varphi^{+}>2 \pi$. Thus, for all $[\rho] e^{i[\varphi]}$ in $S^{*}(\mathbb{C})$, we have $\varphi^{+}-\varphi^{-} \leq 2 \pi, 0 \leq \varphi^{-}<2 \pi$ and $2 \pi<\varphi^{+}<4 \pi$ (see Figure 2). 


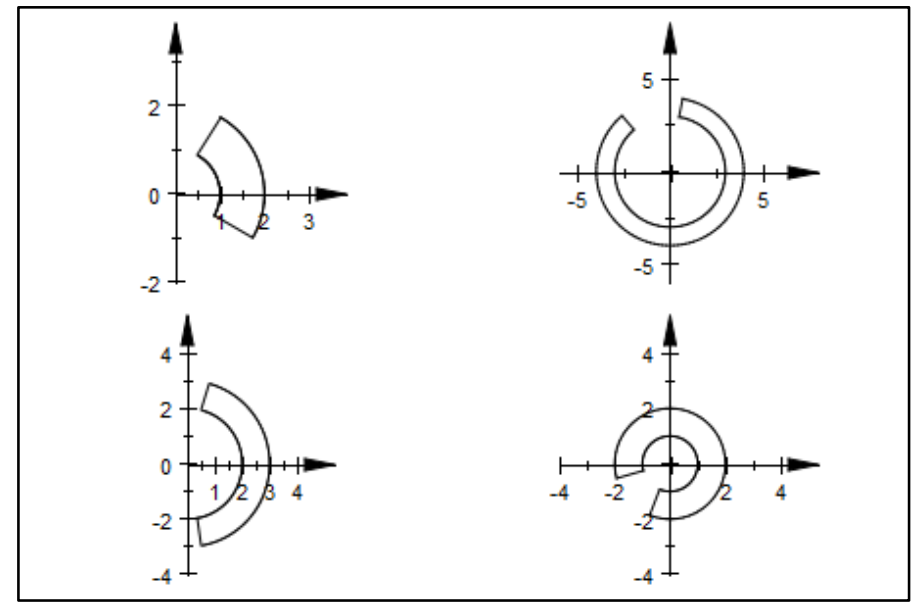

Figure 2. Some members of the set $S^{*}(\mathbb{C})$

\subsection{Operations on Sectors}

Definition 2 Let $S_{1}=\left[\rho_{1}\right] e^{i\left[\theta_{1}\right]}, S_{2}=\left[\rho_{2}\right] e^{i\left[\theta_{2}\right]}$ be two sectors, and $* \in\{+,-, \cdot$,$\} . Then we define the$ corresponding operations for $S_{1}$ and $S_{2}$ by $S_{1} * S_{2}:=(S)\left\{S_{1} \circledast S_{2}\right\}$, where $S_{1} \circledast S_{2}:=\left\{s_{1} * S_{2}: s_{1} \in S_{1}, s_{2} \in S_{2}\right\}$ and $\left(S S_{1} \circledast S_{2}\right\}$ is the smallest sector that encloses $S_{1} \circledast S_{2}$.

Let $S_{1}=\left[\rho_{1}\right] e^{i\left[\theta_{1}\right]}=\left[\rho_{1}^{-}, \rho_{1}^{+}\right] e^{i\left[\theta_{1}^{-}, \theta_{1}^{+}\right]}$and $S_{2}=\left[\rho_{2}\right] e^{i\left[\theta_{2}\right]}=\left[\rho_{2}^{-}, \rho_{2}^{+}\right] e^{i\left[\theta_{2}^{-}, \theta_{2}^{+}\right]}$be given sectors. If the set $S_{1} \circledast S_{2}$ is already a sector, then clearly $(S)\left\{S_{1} \circledast S_{2}\right\}=S_{1} \circledast S_{2}$. Some important properties of $S_{1} \circledast S_{2}$ are summarized below.

1. The results from multiplication $S_{1} \odot S_{2}$ or division $S_{1} \oslash S_{2}$ are sectors. Therefore, $(S)\left\{S_{1} \odot S_{2}\right\}=$ $S_{1} \odot S_{2}$ and $\left(\left\{S_{1} \oslash S_{2}\right\}=S_{1} \oslash S_{2}\right.$.

2. The results from addition $S_{1} \oplus S_{2}$ or subtraction $S_{1} \ominus S_{2}$ are not sectors in general.

For this reason the addition of sectors has been handled with different approaches in the papers $[5,6]$ and [7]. The method in [5] is to transform the sectors into rectangular regions, perform the addition exactly and map the result back into a sector. Obviously this method introduces a serious amount of pessimism and is far from being optimal. The method in [6] is optimal except for some certain cases but with high computational cost. Finally the algorithm introduced in [7] contains some errors as we will show in the sequel.

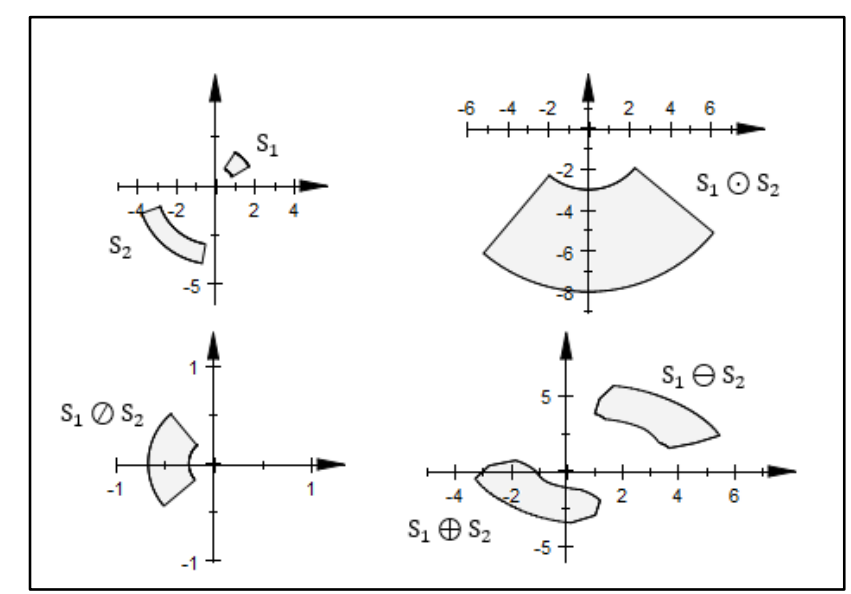

Figure 3. Sectors are not closed under addition and subtraction 
Consider the example, $S_{1}=[1,2] e^{i\left[30^{\circ}, 60^{\circ}\right]}, S_{2}=[3,4] e^{i\left[200^{\circ}, 260^{\circ}\right]}$. Then, the resulting set from $S_{1} \odot S_{2}$ or $S_{1} \oslash S_{2}$ is a sector, while the results from $S_{1} \oplus S_{2}$ or $S_{1} \ominus S_{2}$ are not sectors. This can be seen in Figure 3 .

By the rules for arithmetic operations in $\mathbb{I}(\mathbb{R})$ and the continuity of the exponential function the multiplication and division operations can easily performed as follows:

\section{- Multiplication}

The multiplication of $S_{1}$ and $S_{2}$ is given by,

$$
\begin{aligned}
& S_{1} \cdot S_{2}:=\left(S\left\{S_{1} \odot S_{2}\right\}\right. \\
& =\left(S\left\{\rho_{1} \rho_{2} e^{i\left(\theta_{1}+\theta_{2}\right)}: \rho_{1} \in\left[\rho_{1}\right], \rho_{2} \in\left[\rho_{2}\right], \theta_{1} \in\left[\theta_{1}\right], \theta_{2} \in\left[\theta_{2}\right]\right\}\right. \\
& =\left[\rho_{1}^{-} \rho_{2}^{-}, \rho_{1}^{+} \rho_{2}^{+}\right] e^{i\left[\theta_{1}^{-}+\theta_{2}^{-}, \theta_{1}^{+}+\theta_{2}^{+}\right]} .
\end{aligned}
$$

Note that, from the definition of a sector, when $\left(\theta_{1}^{+}+\theta_{2}^{+}\right)-\left(\theta_{1}^{-}+\theta_{2}^{-}\right) \geq 2 \pi$ we have to set $\left[\theta_{1}^{-}+\theta_{2}^{-}, \theta_{1}^{+}+\theta_{2}^{+}\right]=[0,2 \pi]$.

\section{- Division}

The division of $S_{1}$ and $S_{2}$ is given by,

$$
\begin{aligned}
& S_{1} / S_{2}:=\left(\left\{S_{1} \oslash S_{2}\right\}\right. \\
& \left.\left.\left.\left.=\left(S\left\{\frac{\rho_{1}}{\rho_{2}} e^{i\left(\theta_{1}-\theta_{2}\right)}: \rho_{1} \in \rho_{1}\right], \rho_{2} \in \rho_{2}\right], \theta_{1} \in \theta_{1}\right], \theta_{2} \in \theta_{2}\right]\right\}, 0 \notin \rho_{2}\right] \\
& = \begin{cases}{\left[\frac{\rho_{1}^{-}}{\rho_{2}^{+}}, \frac{\rho_{1}^{+}}{\rho_{2}^{-}}\right] e^{i\left[\theta_{1}^{-}-\theta_{2}^{+}+2 n \pi, \theta_{1}^{+}-\theta_{2}^{-}+2 n \pi\right]}, \text { if } \theta_{1}^{-}-\theta_{2}^{+}<0} \\
{\left[\frac{\rho_{1}^{-}}{\rho_{2}^{+}}, \frac{\rho_{1}^{+}}{\rho_{2}^{-}}\right] e^{i\left[\theta_{1}^{-}-\theta_{2}^{+}, \theta_{1}^{+}-\theta_{2}^{-}\right],} \quad \text { otherwise },}\end{cases}
\end{aligned}
$$

where $n$ is the smallest positive integer such that $\theta_{1}^{-}-\theta_{2}^{+}+2 n \pi \geq 0$. Also we set $\left[\theta_{1}^{-}-\theta_{2}^{+}, \theta_{1}^{+}-\theta_{2}^{-}\right]=[0,2 \pi]$ when $\left(\theta_{1}^{+}-\theta_{2}^{-}\right)-\left(\theta_{1}^{-}-\theta_{2}^{+}\right) \geq 2 \pi$.

\section{ADDITION AND SUBTRACTION OF TWO SECTORS}

In general there is no simple, direct way to add or subtract two sectors $S_{1}$ and $S_{2}$, but the minimal sector, (S) $\left\{S_{1} \circledast S_{2}\right\}$ can be obtained from $S_{1} \circledast S_{2}$. In order to evaluate this hull, we have to compute the best possible lower and upper bounds for both $[\rho]$ and $[\varphi]$ such that the sector they represent is the smallest possible sector that contains the set $S_{1} \circledast S_{2}$.

The problem of subtraction can be transformed into addition. That is subtraction of $S_{1}$ and $S_{2}$ can be calculated as: $S_{1}-S_{2}=S_{1}+\left(-S_{2}\right)$, where $\left(-S_{2}\right):=\left\{w:-w \in S_{2}\right\}$. Therefore, we can use addition to solve subtraction problems as well.

In this section, the basis of a method for determining $\left(S S_{1} \oplus S_{2}\right\}$ will be presented.

Let $s_{1} \in S_{1}, s_{2} \in S_{2}$ and formualte $s_{1}+s_{2}=s$ as:

$$
\rho_{1} e^{i \theta_{1}}+\rho_{2} e^{i \theta_{2}}=\rho e^{i \varphi} .
$$

To determine $\rho$, we simply take the complex absolute value of both sides of Equation (1). Hence, 


$$
\begin{aligned}
\rho^{2} & =\left|\rho_{1} e^{i \theta_{1}}+\rho_{2} e^{i \theta_{2}}\right| \\
& =\rho_{1}^{2}+\rho_{2}^{2}+2 \rho_{1} \rho_{2} \cos \left(\theta_{1}-\theta_{2}\right) .
\end{aligned}
$$

Taking the positive square roots yields,

$$
\rho=\sqrt{\rho_{1}^{2}+\rho_{2}^{2}+2 \rho_{1} \rho_{2} \cos \left(\theta_{1}-\theta_{2}\right)}
$$

To determine $\varphi$, from Equation (1), we have,

$$
\begin{aligned}
& \operatorname{Re}(s)=\rho \cos (\varphi)=\rho_{1} \cos \left(\theta_{1}\right)+\rho_{2} \cos \left(\theta_{2}\right), \\
& \operatorname{Im}(s)=\rho \sin (\varphi)=\rho_{1} \sin \left(\theta_{1}\right)+\rho_{2} \sin \left(\theta_{2}\right) .
\end{aligned}
$$

From the last two equations we get,

$$
\tan (\varphi)=\frac{\rho_{1} \sin \left(\theta_{1}\right)+\rho_{2} \sin \left(\theta_{2}\right)}{\rho_{1} \cos \left(\theta_{1}\right)+\rho_{2} \cos \left(\theta_{2}\right)} .
$$

\subsection{Computing The Bounds of Angle Interval [ $\varphi]$}

Recall that $\tan \varphi$ was given in Equation (3) and thus:

$$
\varphi=\arctan \left(\frac{\rho_{1} \sin \left(\theta_{1}\right)+\rho_{2} \sin \left(\theta_{2}\right)}{\rho_{1} \cos \left(\theta_{1}\right)+\rho_{2} \cos \left(\theta_{2}\right)}\right) .
$$

where $\varphi \in] \frac{-\pi}{2}, \frac{\pi}{2}$ [ and the quadrant in which it falls is determined by the signs of $\rho_{1} \sin \left(\theta_{1}\right)+\rho_{2} \sin \left(\theta_{2}\right)$ and $\rho_{1} \cos \left(\theta_{1}\right)+\rho_{2} \cos \left(\theta_{2}\right)$. If $\rho_{1} \cos \left(\theta_{1}\right)+\rho_{2} \cos \left(\theta_{2}\right)=0$, then

$$
\varphi= \begin{cases}\frac{\pi}{2}, & \text { if } \rho_{1} \sin \left(\theta_{1}\right)+\rho_{2} \sin \left(\theta_{2}\right)>0 \\ \frac{3 \pi}{2}, & \text { if } \rho_{1} \sin \left(\theta_{1}\right)+\rho_{2} \sin \left(\theta_{2}\right)<0\end{cases}
$$

Hence for each $s \in S_{1} \oplus S_{2}$ we can compute the corresponding value of $\varphi$, except when $s=0$.

Our goal is to compute the bounds $\varphi^{-}$and $\varphi^{+}$such that $\left[\varphi^{-}, \varphi^{+}\right]$is the least interval that encloses all possible angles of $S_{1} \oplus S_{2}$. This means that we have to compute the minimum and the maximum of the function $\varphi$ with respect to all points $\left(\rho_{1}, \rho_{2}, \theta_{1}, \theta_{2}\right) \in \Omega=\left[\rho_{1}\right] \times\left[\rho_{2}\right] \times\left[\theta_{1}\right] \times\left[\theta_{2}\right]$.

Differentiating $\varphi$ we obtain:

$$
\begin{aligned}
& \frac{\partial \varphi}{\partial \rho_{1}}=\frac{\rho_{2} \sin \left(\theta_{1}-\theta_{2}\right)}{\rho^{2}}, \\
& \frac{\partial \varphi}{\partial \rho_{2}}=\frac{-\rho_{1} \sin \left(\theta_{1}-\theta_{2}\right)}{\rho^{2}}, \\
& \frac{\partial \varphi}{\partial \theta_{1}}=\frac{\rho_{1} \rho_{2} \cos \left(\theta_{1}-\theta_{2}\right)+\rho_{1}^{2}}{\rho^{2}}, \\
& \frac{\partial^{2} \varphi}{\partial \theta_{1}^{2}}=\frac{\rho_{1} \rho_{2} \sin \left(\theta_{1}-\theta_{2}\right)\left(\rho_{1}^{2}-\rho_{2}^{2}\right)}{\rho^{4}}, \\
& \frac{\partial \varphi}{\partial \theta_{2}}=\frac{\rho_{1} \rho_{2} \cos \left(\theta_{1}-\theta_{2}\right)+\rho_{2}^{2}}{\rho^{2}}, \\
& \frac{\partial^{2} \varphi}{\partial \theta_{2}^{2}}=\frac{\rho_{1} \rho_{2} \sin \left(\theta_{1}-\theta_{2}\right)\left(\rho_{1}^{2}-\rho_{2}^{2}\right)}{\rho^{4}} .
\end{aligned}
$$

Note that $\nabla \varphi\left(\rho_{1}, \rho_{2}, \theta_{1}, \theta_{2}\right)$ is defined whenever $\varphi$ is. Thus, it is easy to check that the function $\varphi$ has no critical points on its domain of definition.

We will find two points $x_{\min }, x_{\max } \in \Omega$ such that, $\min \varphi=\varphi^{-}=\varphi\left(x_{\min }\right) \leq \varphi(x) \leq \varphi\left(x_{\max }\right)=\varphi^{+}=\max \varphi$ for all $x \in \Omega$. 
From $\frac{\partial \varphi}{\partial \rho_{1}}$ and $\frac{\partial \varphi}{\partial \rho_{2}}$ we note that the possible values of $\rho_{1}$ and $\rho_{2}$ are $\left\{\rho_{1}^{+}, \rho_{1}^{-}\right\}$and $\left\{\rho_{2}^{+}, \rho_{2}^{-}\right\}$, respectively. Hence the optimal point $\left(x_{\min }\right.$ or $\left.x_{\max }\right)$ must fall into one of the following types of points:

- $X_{1}=\left\{\left(\rho_{1}, \rho_{2}, \theta_{1}, \theta_{2}\right): \rho_{1} \in\left\{\rho_{1}^{+}, \rho_{1}^{-}\right\}, \rho_{2} \in\left\{\rho_{2}^{+}, \rho_{2}^{-}\right\}, \theta_{1} \in\right] \theta_{1}\left[, \theta_{2} \in\left\{\theta_{2}^{+}, \theta_{2}^{-}\right\}\right\}$, where $\theta_{1}$ is given by solving the equation $\frac{\partial \varphi}{\partial \theta_{1}}=0$.

- $X_{2}=\left\{\left(\rho_{1}, \rho_{2}, \theta_{1}, \theta_{2}\right): \rho_{1} \in\left\{\rho_{1}^{+}, \rho_{1}^{-}\right\}, \rho_{2} \in\left\{\rho_{2}^{+}, \rho_{2}^{-}\right\}, \theta_{1} \in\left\{\theta_{1}^{+}, \theta_{1}^{-}\right\}, \theta_{2} \in\right] \theta_{2}[\}$, where $\theta_{2}$ is given by solving the equation $\frac{\partial \varphi}{\partial \theta_{2}}=0$.

- $X_{3}=\left\{\left(\rho_{1}, \rho_{2}, \theta_{1}, \theta_{2}\right): \rho_{1} \in\left\{\rho_{1}^{+}, \rho_{1}^{-}\right\}, \rho_{2} \in\left\{\rho_{2}^{+}, \rho_{2}^{-}\right\}, \theta_{1} \in\left\{\theta_{1}^{+}, \theta_{1}^{-}\right\}, \theta_{2} \in\left\{\theta_{2}^{+}, \theta_{2}^{-}\right\}\right\}$. Notice that we only need to select $\theta_{1}$ and $\theta_{2}$ because the signs of $\frac{\partial \varphi}{\partial \rho_{1}}$ and $\frac{\partial \varphi}{\partial \rho_{2}}$ are determined by the $\operatorname{sign}$ of $\sin \left(\theta_{1}-\theta_{2}\right)$. In what follows we identify the optimality conditions of $\theta_{1}$ and $\theta_{2}$.

\section{Minimality condition:}

$\theta_{1}=\left\{\begin{array}{ll}\theta_{1}^{-}, & \text {if } \rho_{1} \rho_{2} \cos \left(\theta_{1}^{-}-\theta_{2}\right)+\rho_{1}^{2} \geq 0, \\ \theta_{1}^{+}, & \text {if } \rho_{1} \rho_{2} \cos \left(\theta_{1}^{+}-\theta_{2}\right)+\rho_{1}^{2}<0\end{array}, \theta_{2}= \begin{cases}\theta_{2}^{-}, & \text {if } \rho_{1} \rho_{2} \cos \left(\theta_{1}-\theta_{2}^{-}\right)+\rho_{2}^{2} \geq 0, \\ \theta_{2}^{+}, & \text {if } \rho_{1} \rho_{2} \cos \left(\theta_{1}-\theta_{2}^{+}\right)+\rho_{2}^{2}<0 .\end{cases}\right.$

\section{Maximality condition:}

$\theta_{1}=\left\{\begin{array}{ll}\theta_{1}^{+}, & \text {if } \rho_{1} \rho_{2} \cos \left(\theta_{1}^{+}-\theta_{2}\right)+\rho_{1}^{2} \geq 0, \\ \theta_{1}^{-}, & \text {if } \rho_{1} \rho_{2} \cos \left(\theta_{1}^{-}-\theta_{2}\right)+\rho_{1}^{2}<0\end{array}, \theta_{2}= \begin{cases}\theta_{2}^{+} & \text {if } \rho_{1} \rho_{2} \cos \left(\theta_{1}-\theta_{2}^{+}\right)+\rho_{2}^{2} \geq 0, \\ \theta_{2}^{-} & \text {if } \rho_{1} \rho_{2} \cos \left(\theta_{1}-\theta_{2}^{-}\right)+\rho_{2}^{2}<0 .\end{cases}\right.$

Without loss of generality, we will assume that $\rho_{1}^{-} \leq \rho_{2}^{-}$. We write $\operatorname{int}\left(S_{1} \oplus S_{2}\right)$ and $\partial\left(S_{1} \oplus S_{2}\right)$ to denote the interior of $S_{1} \oplus S_{2}$ and the boundary of $S_{1} \oplus S_{2}$, respectively and split the problem into two cases.

Case 1. $\left.\left[\rho_{1}\right] \cap \rho_{2}\right]=\emptyset$.

In this case neither $0 \in \operatorname{int}\left(S_{1} \oplus S_{2}\right)$ nor $0 \in \partial\left(S_{1} \oplus S_{2}\right)$, i.e., $0 \notin S_{1} \oplus S_{2}$. Also, since $\rho_{1}<\rho_{2}$ for all $\rho_{1} \in\left[\rho_{1}\right], \rho_{2} \in\left[\rho_{2}\right]$, we have $X_{2}=\varnothing$ because $\frac{\partial \varphi}{\partial \theta_{2}} \neq 0$ for any $\left(\rho_{1}, \rho_{2}, \theta_{1}, \theta_{2}\right) \in \Omega$.

In fact we always have $\frac{\partial \varphi}{\partial \theta_{2}}>0$, and accordingly $\varphi$ reaches its minimum (maximum) value when $\theta_{2}$ is minimum (maximum). Therefore we have

$$
\begin{aligned}
& \varphi^{-}=\min \varphi\left(\rho_{1}, \rho_{2}, \theta_{1}, \theta_{2}^{-}\right), \\
& \varphi^{+}=\max \varphi\left(\rho_{1}, \rho_{2}, \theta_{1}, \theta_{2}^{+}\right) .
\end{aligned}
$$

Hence the optimal point falls in $X_{1}$ or $X_{3}$ and rules out $X_{2}$.

\section{Points of type $X_{1}$}

Suppose that $\left[\theta_{1}\right]$ is not a degenerate interval (i.e., $\theta_{1}^{+}>\theta_{1}^{-}$). We have $\frac{\partial \varphi}{\partial \theta_{1}}=0$ when, $\rho_{1} \rho_{2} \cos \left(\theta_{1}-\theta_{2}\right)+\rho_{1}^{2}=0$, from which it follows that $\cos \left(\theta_{1}-\theta_{2}\right)=\frac{-\rho_{1}}{\rho_{2}}$. Using the fact that, $\sin ^{2}+\cos ^{2}=1$, we get $\sin \left(\theta_{1}-\theta_{2}\right)= \pm \sqrt{1-\left(\frac{\rho_{1}}{\rho_{2}}\right)^{2}}$.

Since $\frac{\partial^{2} \varphi}{\partial \theta_{1}^{2}}>0$ when $\sin \left(\theta_{1}-\theta_{2}\right)<0$ and $\frac{\partial^{2} \varphi}{\partial \theta_{1}^{2}}<0$ when $\sin \left(\theta_{1}-\theta_{2}\right)>0$, it follows that $\varphi$ is 
minimum (maximum) when $\sin \left(\theta_{1}-\theta_{2}\right)<0\left(\sin \left(\theta_{1}-\theta_{2}\right)>0\right)$.

Thus, since $\frac{\partial \varphi}{\partial \rho_{1}}<0$ and $\frac{\partial \varphi}{\partial \rho_{2}}>0$ when $\sin \left(\theta_{1}-\theta_{2}\right)<0$, we conclude that the minimum of $\varphi$, among all points of $X_{1}$, is attainable at the point $x_{1 \mathrm{~min}}=\left(\rho_{1}^{+}, \rho_{2}^{-}, \theta_{1}, \theta_{2}^{-}\right)$if $\sin \left(\theta_{1}-\theta_{2}^{-}\right)<0$, where $\theta_{1}$ can be determined as follows

$$
\begin{aligned}
& \theta_{1}-\theta_{2}^{-}=\arctan \left(\frac{\sin \left(\theta_{1}-\theta_{2}^{-}\right)}{\cos \left(\theta_{1}-\theta_{2}^{-}\right)}\right)+\pi \\
& =\arctan \left(\frac{-\sqrt{1-\left(\frac{\rho_{1}^{+}}{\rho_{2}^{-}}\right)^{2}}}{-\frac{\rho_{1}^{+}}{\rho_{2}^{-}}}\right)+\pi
\end{aligned}
$$

i.e,

$$
\theta_{1}=\theta_{2}^{-}+\arctan \left(\frac{\sqrt{\left(\rho_{2}^{-}\right)^{2}-\left(\rho_{1}^{+}\right)^{2}}}{\rho_{1}^{+}}\right)+\pi \pm 2 \pi k
$$

where $k \in\{0,1\}$ (Throughout the rest of the paper $k$ will be in $\{0,1\}$ ).

Similarly, assuming $\theta_{2}=\theta_{2}^{+}$, the maximum of $\varphi$, among all points of $X_{1}$, is attainable at the point $x_{1 \text { max }}=\left(\rho_{1}^{+}, \rho_{2}^{-}, \theta_{1}, \theta_{2}^{+}\right)$if $\sin \left(\theta_{1}-\theta_{2}^{+}\right)>0$, where $\theta_{1}$ is given by

$$
\theta_{1}=\theta_{2}^{+}-\arctan \left(\frac{\sqrt{\left(\rho_{2}^{-}\right)^{2}-\left(\rho_{1}^{+}\right)^{2}}}{\rho_{1}^{+}}\right)+\pi \pm 2 \pi k
$$

The above analysis can be summarized as follows.

1. If there exists $\left.\theta_{1} \in\right] \theta_{1}\left[\right.$ such that $\cos \left(\theta_{1}-\theta_{2}^{-}\right)=\frac{-\rho_{1}^{+}}{\rho_{2}^{-}}$and $\sin \left(\theta_{1}-\theta_{2}^{-}\right)<0$, then, $\varphi\left(x_{1 \min }\right) \leq \varphi\left(x_{1}\right)$ for all $x_{1} \in X_{1}$.

2. If there exists $\left.\theta_{1} \in\right] \theta_{1}\left[\right.$ such that $\cos \left(\theta_{1}-\theta_{2}^{+}\right)=\frac{-\rho_{1}^{+}}{\rho_{2}^{-}}$and $\sin \left(\theta_{1}-\theta_{2}^{+}\right)>0$, then, $\varphi\left(x_{1 \max }\right) \geq \varphi\left(x_{1}\right)$ for all $x_{1} \in X_{1}$.

\section{Points of type $X_{3}$}

In general, $X_{3}$ contains 16 points. Among these 16 points, we will determine the pair $x_{3 \min }$ and $x_{3 \max }$ such that $\varphi\left(x_{3 \min }\right) \leq \varphi\left(x_{3}\right) \leq \varphi\left(x_{3 \max }\right)$ for all $x_{3} \in X_{3}$.

To find the point $x_{3 \min }$, recall that $\varphi^{-}=\min \varphi\left(\rho_{1}, \rho_{2}, \theta_{1}, \theta_{2}^{-}\right)$. Since $\theta_{1}$ has two possible values (i.e., $\left.\theta_{1} \in\left\{\theta_{1}^{+}, \theta_{1}^{-}\right\}\right)$, we have

$$
x_{3 \min } \in\left\{\left(\rho_{1}, \rho_{2}, \theta_{1}^{-}, \theta_{2}^{-}\right),\left(\rho_{1}, \rho_{2}, \theta_{1}^{+}, \theta_{2}^{-}\right)\right\},
$$

where at least one of $\theta_{1}^{+}$and $\theta_{1}^{-}$must achieve the minimality condition. If both $\theta_{1}^{+}$and $\theta_{1}^{-}$achieve the minimality condition, then,

where

$$
x_{3 \min }= \begin{cases}\left(\rho_{1}, \rho_{2}, \theta_{1}^{-}, \theta_{2}^{-}\right), & \text {if } \sin \left(\theta_{1}^{-}-\theta_{2}^{-}\right)<0 \text { and } \sin \left(\theta_{1}^{+}-\theta_{2}^{-}\right) \geq 0, \\ \left(\rho_{1}, \rho_{2}, \theta_{1}^{+}, \theta_{2}^{-}\right), & \text {if } \sin \left(\theta_{1}^{+}-\theta_{2}^{-}\right)<0 \text { and } \sin \left(\theta_{1}^{-}-\theta_{2}^{-}\right) \geq 0, \\ \min \left\{\left(\rho_{1}, \rho_{2}, \theta_{1}^{-}, \theta_{2}^{-}\right),\left(\rho_{1}, \rho_{2}, \theta_{1}^{+}, \theta_{2}^{-}\right)\right\}, & \text {otherwise }\end{cases}
$$

$$
\left\{\begin{array}{l}
\rho_{1}=\rho_{1}^{+}, \rho_{2}=\rho_{2}^{-}, \text {if } \sin \left(\theta_{1}-\theta_{2}^{-}\right) \leq 0, \\
\rho_{1}=\rho_{1}^{-}, \rho_{2}=\rho_{2}^{+} \text {otherwise. }
\end{array}\right.
$$


Similarly, by fixing $\theta_{2}$ at $\theta_{2}^{+}$, we have, $x_{3 \max } \in\left\{\left(\rho_{1}, \rho_{2}, \theta_{1}^{-}, \theta_{2}^{+}\right),\left(\rho_{1}, \rho_{2}, \theta_{1}^{+}, \theta_{2}^{+}\right)\right\}$, where $\theta_{1} \in\left\{\theta_{1}^{+}, \theta_{1}^{-}\right\}$ is determined according to the maximality condition. If both $\theta_{1}^{+}$and $\theta_{1}^{-}$achieve the maximality condition, then,

where

$$
x_{3 \max }= \begin{cases}\left(\rho_{1}, \rho_{2}, \theta_{1}^{-}, \theta_{2}^{+}\right), & \text {if } \sin \left(\theta_{1}^{-}-\theta_{2}^{+}\right)>0 \text { and } \sin \left(\theta_{1}^{+}-\theta_{2}^{+}\right) \leq 0, \\ \left(\rho_{1}, \rho_{2}, \theta_{1}^{+}, \theta_{2}^{+}\right), & \text {if } \sin \left(\theta_{1}^{+}-\theta_{2}^{+}\right)>0 \text { and } \sin \left(\theta_{1}^{-}-\theta_{2}^{+}\right) \leq 0, \\ \min \left\{\left(\rho_{1}, \rho_{2}, \theta_{1}^{-}, \theta_{2}^{+}\right),\left(\rho_{1}, \rho_{2}, \theta_{1}^{+}, \theta_{2}^{+}\right)\right\} & \text {otherwise }\end{cases}
$$

$$
\left\{\begin{array}{l}
\rho_{1}=\rho_{1}^{+}, \rho_{2}=\rho_{2}^{-}, \text {if } \sin \left(\theta_{1}-\theta_{2}^{+}\right) \geq 0, \\
\rho_{1}=\rho_{1}^{-}, \rho_{2}=\rho_{2}^{+}, \text {otherwise. }
\end{array}\right.
$$

\section{Special subcase of Case1}

There remains a case in which the optimal bounds of $\varphi$ can not be determined in the usual ways. Figure 4 demonstrates two examples.

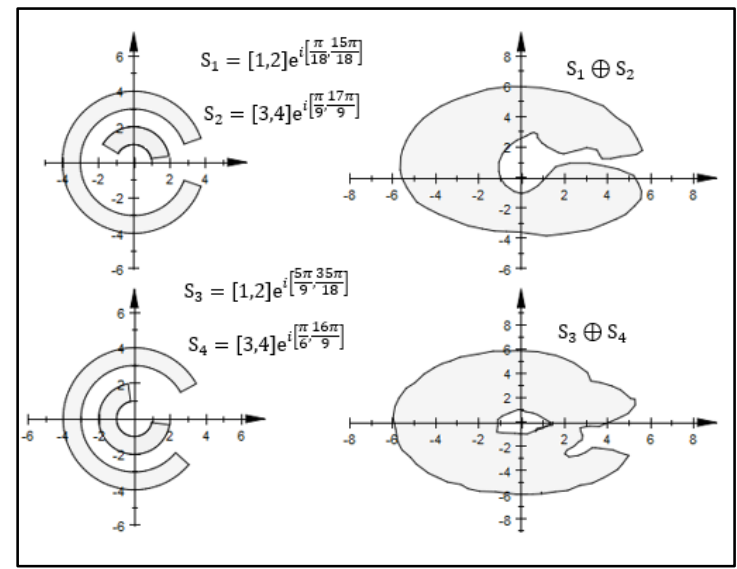

Figure 4. Case of annulus

It is clear that any sector, which encloses the set $S_{1} \oplus S_{2}$ or $S_{3} \oplus S_{4}$ is an annulus and has an angle interval $\left[\varphi^{-}, \varphi^{+}\right]=[0,2 \pi]$. In this special case, one can check that one of the following holds:

- $\left(\rho_{1}, \rho_{2}, \theta_{1}^{-}, \theta_{2}^{-}\right)$and $\left(\rho_{1}, \rho_{2}, \theta_{1}^{+}, \theta_{2}^{-}\right)$are both candidates for $\varphi^{-}$with, $\left|\varphi\left(\rho_{1}, \rho_{2}, \theta_{1}^{-}, \theta_{2}^{-}\right)-\varphi\left(\rho_{1}, \rho_{2}, \theta_{1}^{+}, \theta_{2}^{-}\right)\right|>\pi$,

- $\left(\rho_{1}, \rho_{2}, \theta_{1}^{-}, \theta_{2}^{+}\right)$and $\left(\rho_{1}, \rho_{2}, \theta_{1}^{+}, \theta_{2}^{+}\right)$are both candidates for $\varphi^{+}$with, $\left|\varphi\left(\rho_{1}, \rho_{2}, \theta_{1}^{-}, \theta_{2}^{+}\right)-\varphi\left(\rho_{1}, \rho_{2}, \theta_{1}^{+}, \theta_{2}^{+}\right)\right|>\pi$,

$$
\cdot \varphi^{+}-\varphi^{-}<\frac{\theta_{2}^{+}-\theta_{2}^{-}}{2}
$$

Case 2. $\left[\rho_{1}\right] \cap\left[\rho_{2}\right] \neq \varnothing$.

In this case it may happen that $\rho_{1} \cos \left(\theta_{1}\right)+\rho_{2} \cos \left(\theta_{2}\right)=0$ and $\rho_{1} \sin \left(\theta_{1}\right)+\rho_{2} \sin \left(\theta_{2}\right)=0$. This clearly happens when either $\rho_{1}=\rho_{2}=0$; or $\rho_{1}=\rho_{2}$ and $\theta_{1}-\theta_{2}= \pm(2 k+1) \pi$. When this happens, we have either $0 \in \partial\left(S_{1} \oplus S_{2}\right)$ or $0 \in \operatorname{int}\left(S_{1} \oplus S_{2}\right)$. The case of $0 \in \operatorname{int}\left(S_{1} \oplus S_{2}\right)$ needs special attention because $\varphi^{-}$and $\varphi^{+}$cannot be determined in the usual way. That $0 \in \operatorname{int}\left(S_{1} \oplus S_{2}\right)$ occurs in the following two situations.

1. $\left.\left[\rho_{1}\right] \cap \rho_{2}\right]=\rho_{1}^{+}=\rho_{2}^{-}$and both $\left.-(2 k+1) \pi \in\right] \theta_{1}^{-}-\theta_{2}^{+}, \theta_{1}^{+}-\theta_{2}^{-}[$and 
$(2 k+1) \pi \in] \theta_{1}^{-}-\theta_{2}^{+}, \theta_{1}^{+}-\theta_{2}^{-}\left[\right.$provided that $\left[\rho_{1}\right]$ and $\left[\rho_{2}\right]$ are not both degenerate (thin) intervals. Figure 5 shows two examples.

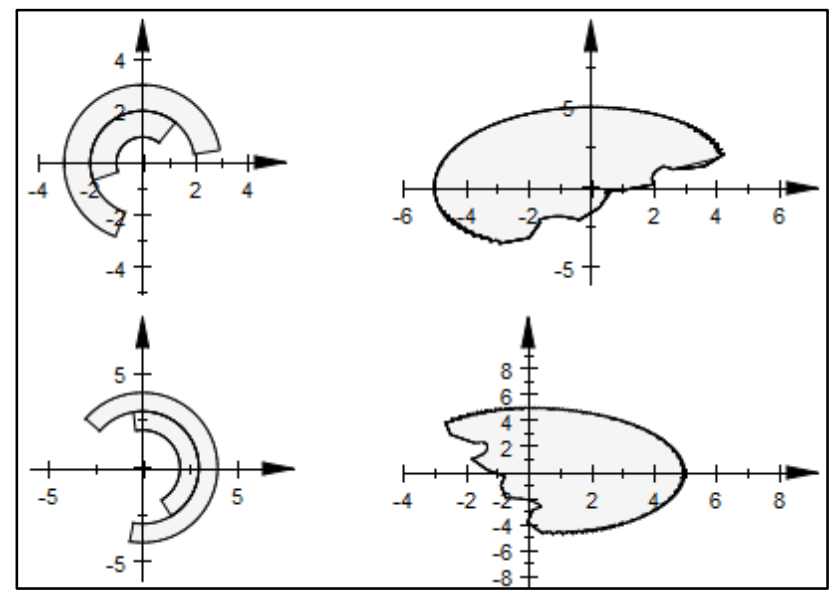

Figure 5. The origin as an interior point

2. $] \rho_{1}[\cap] \rho_{2}[\neq \emptyset$ and either $-(2 k+1) \pi \in] \theta_{1}^{-}-\theta_{2}^{+}, \theta_{1}^{+}-\theta_{2}^{-}[$or $(2 k+1) \pi \in] \theta_{1}^{-}-\theta_{2}^{+}, \theta_{1}^{+}-\theta_{2}^{-}[$. It is obvious that under these conditions $\varphi^{-}=0, \varphi^{+}=2 \pi$.

Now, we proceed to determine $\varphi^{-}$and $\varphi^{+}$in cases where $0 \notin \operatorname{int}\left(S_{1} \oplus S_{2}\right)$. We consider the following two subcases.

Subcase 2.1. $\rho_{1}^{+}=\rho_{2}^{-}$.

Similar to Case 1 , we have $\frac{\partial \varphi}{\partial \theta_{2}}>0$ for any $\left(\rho_{1}, \rho_{2}, \theta_{1}, \theta_{2}\right)$ in the domain of $\varphi$. Therefore,

$$
\begin{aligned}
& \varphi^{-}=\min \varphi\left(\rho_{1}, \rho_{2}, \theta_{1}, \theta_{2}^{-}\right) \\
& \varphi^{+}=\max \varphi\left(\rho_{1}, \rho_{2}, \theta_{1}, \theta_{2}^{+}\right) .
\end{aligned}
$$

We distinguish two cases: $0 \in \partial\left(S_{1} \oplus S_{2}\right)$ and $0 \notin \partial\left(S_{1} \oplus S_{2}\right)$.

$\cdot 0 \in \partial\left(S_{1} \oplus S_{2}\right)$.

This case occurs when either $\left.-(2 k+1) \pi \in \theta_{1}^{-}-\theta_{2}^{+}, \theta_{1}^{+}-\theta_{2}^{-}\right]$or $\left.(2 k+1) \pi \in \theta_{1}^{-}-\theta_{2}^{+}, \theta_{1}^{+}-\theta_{2}^{-}\right]$. Figure 6 shows two examples.

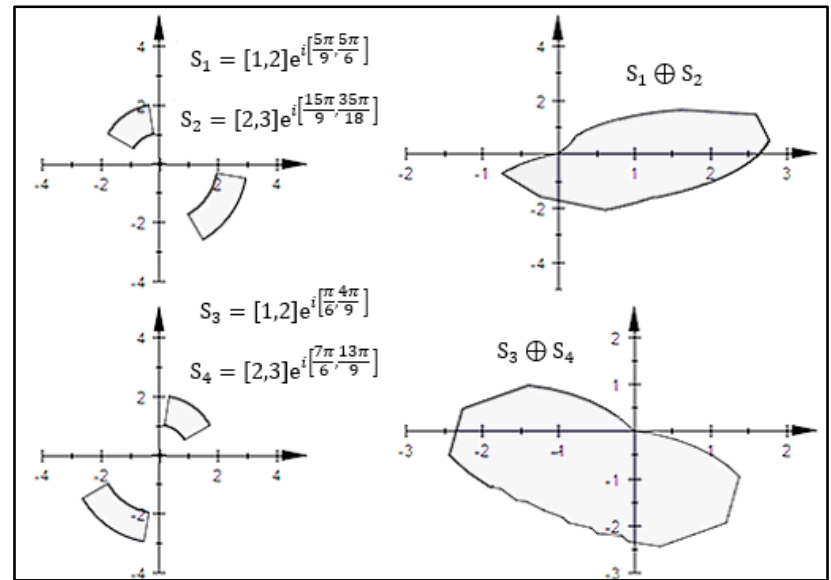

Figure 6. The origin as a boundary point 
Notice that $\varphi$ is undefined at $x=\left(\rho_{1}^{+}, \rho_{2}^{-}, \theta_{1}, \theta_{2}\right)$ when $\theta_{1}-\theta_{2}= \pm(2 k+1) \pi$. Let us investigate how $\varphi$ behaves near the point $x$. Consider the function

$$
h_{1}\left(\theta_{1}, \theta_{2}\right)=\varphi\left(\rho_{1}^{+}, \rho_{2}^{-}, \theta_{1}, \theta_{2}\right)=\arctan \left(\frac{\sin \left(\theta_{1}\right)+\sin \left(\theta_{2}\right)}{\cos \left(\theta_{1}\right)+\cos \left(\theta_{2}\right)}\right),
$$

where $\theta_{1} \in\left[\theta_{1}\right]$ and $\theta_{2} \in\left[\theta_{2}\right]$.

We observe that the function $h_{1}$ reaches its minimum value when $\left|\theta_{1}-\theta_{2}\right|$ approaches $\pi$ from the righthand side, and it reaches its maximum value when $\left|\theta_{1}-\theta_{2}\right|$ approaches $\pi$ from the left-hand side.

Assuming that $\theta_{2}=\theta_{2}^{-}$we compute $\varphi^{-}$as follows. If there exists $\left.\theta_{1} \in\right] \theta_{1}$ [ such that, $\theta_{1}^{-}=\theta_{2}^{-} \pm(2 k+1) \pi$, then $\sin \left(\theta_{1}-\theta_{2}^{-}\right)<0$, which makes $\frac{\partial \varphi}{\partial \rho_{1}}<0$ and $\frac{\partial \varphi}{\partial \rho_{2}}>0$, we compute the minimum of $\varphi$ using $\rho_{1}=\rho_{1}^{+}$and $\rho_{2}=\rho_{2}^{-}$. That is $\varphi$ achieves its minimum at the point, $x_{1 \min }=\left(\rho_{1}^{+}, \rho_{2}^{-}, \theta_{1}, \theta_{2}^{-}\right) \in X_{1}$, i.e.,

$$
\varphi^{-}=\varphi\left(\rho_{1}^{+}, \rho_{2}^{-}, \theta_{1}, \theta_{2}^{-}\right)=\arctan \left(\frac{\sin \left(\theta_{1}\right)+\sin \left(\theta_{2}^{-}\right)}{\cos \left(\theta_{1}\right)+\cos \left(\theta_{2}^{-}\right)}\right) .
$$

If $\left.\theta_{2}^{-}+\epsilon \pm(2 k+1) \pi \notin\right] \theta_{1}\left[\right.$ then clearly $\varphi$ achieves its minimum at $x_{3 \min } \in$ $\left\{\left(\rho_{1}, \rho_{2}, \theta_{1}^{-}, \theta_{2}^{-}\right),\left(\rho_{1}, \rho_{2}, \theta_{1}^{+}, \theta_{2}^{-}\right)\right\} \subset X_{3}$, where $x_{3 \min }$ is selected as in Case 1 provided that $\rho_{1}=\rho_{1}^{-}$and $\rho_{2}=\rho_{2}^{+}$when $\theta_{1}^{+}-\theta_{2}^{-}= \pm(2 k+1) \pi$.

Let us now compute $\varphi^{+}$. If there exists $\left.\theta_{1} \in\right] \theta_{1}$ [ such that $\theta_{1}=\theta_{2}^{+}-\epsilon \pm(2 k+1) \pi$, then, $\sin \left(\theta_{1}-\theta_{2}^{+}\right)>0$, which makes $\frac{\partial \varphi}{\partial \rho_{1}}>0$ and $\frac{\partial \varphi}{\partial \rho_{2}}<0$, we compute the maximum of $\varphi$ using $\rho_{1}=\rho_{1}^{+}$ and $\rho_{2}=\rho_{2}^{-}$. That is $\varphi$ achieves its maximum at the point $x_{1 \max }=\left(\rho_{1}^{+}, \rho_{2}^{-}, \theta_{1}, \theta_{2}^{+}\right) \in X_{1}$, i.e.,

$$
\varphi^{+}=\varphi\left(\rho_{1}^{+}, \rho_{2}^{-}, \theta_{1}, \theta_{2}^{+}\right)=\arctan \left(\frac{\sin \left(\theta_{1}\right)+\sin \left(\theta_{2}^{+}\right)}{\cos \left(\theta_{1}\right)+\cos \left(\theta_{2}^{+}\right)}\right) .
$$

If $\left.\theta_{2}^{+}-\epsilon \pm(2 k+1) \pi \notin\right] \theta_{1}[$, then $\varphi$ achieves its maximum at,

$x_{3 \max } \in\left\{\left(\rho_{1}, \rho_{2}, \theta_{1}^{-}, \theta_{2}^{+}\right),\left(\rho_{1}, \rho_{2}, \theta_{1}^{+}, \theta_{2}^{+}\right)\right\} \subset X_{3}$, where $x_{3 \max }$ is selected as in Case 1 provided that $\rho_{1}=\rho_{1}^{-}$and $\rho_{2}=\rho_{2}^{+}$when $\theta_{1}^{-}-\theta_{2}^{+}= \pm(2 k+1) \pi$.

We end this case with the following remark.

Remark 3 After determining $\varphi^{-}$and $\varphi^{+}$, we have to check whether $\varphi^{+}-\varphi^{-}<\frac{\theta_{2}^{+}-\theta_{2}^{-}}{2}$. If that is the case, we set $\varphi^{-}=0, \varphi^{+}=2 \pi$.

- $0 \notin \partial\left(S_{1} \oplus S_{2}\right)$.

This case occurs when $\theta_{1}-\theta_{2} \neq \pm(2 k+1) \pi$. Consequently,

$$
\begin{aligned}
& \varphi^{-}=\varphi\left(x_{3 \min }\right), \\
& \varphi^{+}=\varphi\left(x_{3 \max }\right) .
\end{aligned}
$$

In other words, $\varphi$ achieves its maximum and minimum at points of type $X_{3}$. In order to prove this, suppose that $X_{1} \neq \varnothing$ with $x_{1 \text { min }}, x_{1 \text { max }} \in X_{1}$ such that $\varphi\left(x_{1 \text { min }}\right) \leq \varphi\left(x_{1}\right) \leq \varphi\left(x_{1 \text { max }}\right)$ for all $x_{1} \in X_{1}$. We want to show that

$$
\begin{aligned}
& \varphi\left(x_{3 \min }\right)<\varphi\left(x_{1 \min }\right), \\
& \varphi\left(x_{3 \max }\right)>\varphi\left(x_{1 \max }\right) .
\end{aligned}
$$


To prove $\varphi\left(x_{3 \min }\right)<\varphi\left(x_{1 \mathrm{~min}}\right)$, we have $x_{1 \min }=\left(\rho_{1}, \rho_{2}, \alpha, \theta_{2}^{-}\right)$, where $\left.\alpha \in\right] \theta_{1}[$ such that, $\cos \left(\alpha-\theta_{2}^{-}\right)=\frac{-\rho_{1}}{\rho_{2}}$. If $\sin \left(\alpha-\theta_{2}^{-}\right)<0$, then $\frac{\partial \varphi}{\partial \rho_{1}}<0$ and $\frac{\partial \varphi}{\partial \rho_{2}}>0$, i.e., $x_{1 \min }=\left(\rho_{1}^{+}, \rho_{2}^{-}, \alpha, \theta_{2}^{-}\right)$. But such a point does not exist in the domain of $\varphi$. If $\sin \left(\alpha-\theta_{2}^{-}\right)>0$ we have $\frac{\partial \varphi}{\partial \rho_{1}}>0$ and $\frac{\partial \varphi}{\partial \rho_{2}}<0$, i.e., $x_{1 \min }=\left(\rho_{1}^{-}, \rho_{2}^{+}, \alpha, \theta_{2}^{-}\right)$. Consider the function,

$$
h_{2}\left(\theta_{1}\right)=\varphi\left(\rho_{1}^{-}, \rho_{2}^{+}, \theta_{1}, \theta_{2}^{-}\right)=\arctan \left(\frac{\rho_{1}^{-} \sin \left(\theta_{1}\right)+\rho_{2}^{+} \sin \left(\theta_{2}^{-}\right)}{\rho_{1}^{-} \cos \left(\theta_{1}\right)+\rho_{2}^{+} \cos \left(\theta_{2}^{-}\right)}\right) .
$$

Considering the second derivative, we see that for $\sin \left(\alpha-\theta_{2}^{-}\right)>0$ we have

$$
\frac{\partial^{2} h_{2}}{\partial \theta_{1}^{2}}=\frac{\partial^{2} \varphi\left(x_{1 \min }\right)}{\partial \theta_{1}^{2}}<0 .
$$

So the graph of $h_{2}$ is concave downward about $\theta_{1}=\alpha$. Therefore, based on the above discussion we conclude that the minimum of $h_{2}$ achieves at one of the end points of [ $\left.\theta_{1}\right]$. Consequently we have $\varphi\left(x_{3 \min }\right)<\varphi\left(x_{1 \min }\right)$. In the same manner, we can show that $\varphi\left(x_{3 \max }\right)>\varphi\left(x_{1 \max }\right)$.

Subcase 2.2. $] \rho_{1}[\cap] \rho_{2}[\neq \varnothing$.

In this case we have $\rho_{1}^{+}>\rho_{2}^{-}$and the only places where $\varphi$ can have extreme values are points of type $X_{3}$, or in other words

$$
\varphi\left(x_{3 \min }\right)<\left\{\begin{array}{ll}
\varphi\left(x_{1}\right), & \text { for all } x_{1} \in X_{1}, \\
\varphi\left(x_{2}\right), & \text { for all } x_{2} \in X_{2},
\end{array} \text { and } \varphi\left(x_{3 \max }\right)> \begin{cases}\varphi\left(x_{1}\right), & \text { for all } x_{1} \in X_{1} \\
\varphi\left(x_{2}\right), & \text { for all } x_{2} \in X_{2}\end{cases}\right.
$$

To see that $\varphi\left(x_{3 \min }\right)<\varphi\left(x_{1}\right)$, suppose that there exists $\left.\alpha \in\right] \theta_{1}\left[\right.$ such that $\cos \left(\alpha-\theta_{2}\right)=\frac{-\rho_{1}}{\rho_{2}}$.

If $\sin \left(\alpha-\theta_{2}\right)>0$, then $x_{1}=\left(\rho_{1}^{-}, \rho_{2}^{+}, \alpha, \theta_{2}\right)$. So $\frac{\partial^{2} \varphi\left(x_{1}\right)}{\partial \theta_{1}^{2}}<0$, showing that $\varphi\left(x_{3 \min }\right)<\varphi\left(x_{1}\right)$. Now suppose that $\sin \left(\alpha-\theta_{2}\right)<0$, then $\rho_{1}=\rho_{1}^{+}$and $\rho_{2}=\rho_{2}^{-}$. It follows that $\cos \left(\alpha-\theta_{2}\right)=\frac{-\rho_{1}^{+}}{\rho_{2}^{-}}<-1$, which is impossible.

To see that $\varphi\left(x_{3 \max }\right)>\varphi\left(x_{1}\right)$, suppose that there exists $\left.\beta \in\right] \theta_{1}\left[\right.$ such that $\cos \left(\beta-\theta_{2}\right)=\frac{-\rho_{1}}{\rho_{2}}$. If $\sin \left(\beta-\theta_{2}\right)<0$, then $x_{1}=\left(\rho_{1}^{-}, \rho_{2}^{+}, \beta, \theta_{2}\right)$. So $\frac{\partial^{2} \varphi\left(x_{1}\right)}{\partial \theta_{1}^{2}}>0$, showing that $\varphi\left(x_{3 \max }\right)>\varphi\left(x_{1}\right)$.

If $\sin \left(\beta-\theta_{2}\right)>0$, we have $\cos \left(\beta-\theta_{2}\right)=\frac{-\rho_{1}^{+}}{\rho_{2}^{-}}<-1$, which is impossible.

In the same way we can show that $\varphi\left(x_{3 \min }\right)<\varphi\left(x_{2}\right)$ and $\varphi\left(x_{3 \max }\right)>\varphi\left(x_{2}\right)$ for any $x_{2} \in X_{2}$. Thus to compute $\varphi^{-}$and $\varphi^{+}$, it is sufficient to find $x_{3 \min }$ and $x_{3 \max }$. We have,

$$
\begin{aligned}
& x_{3 \min } \in\left\{\left(\rho_{1}, \rho_{2}, \theta_{1}^{-}, \theta_{2}^{+}\right),\left(\rho_{1}, \rho_{2}, \theta_{1}^{-}, \theta_{2}^{-}\right),\left(\rho_{1}, \rho_{2}, \theta_{1}^{+}, \theta_{2}^{-}\right)\right\}, \\
& x_{3 \max } \in\left\{\left(\rho_{1}, \rho_{2}, \theta_{1}^{-}, \theta_{2}^{+}\right),\left(\rho_{1}, \rho_{2}, \theta_{1}^{+}, \theta_{2}^{+}\right),\left(\rho_{1}, \rho_{2}, \theta_{1}^{+}, \theta_{2}^{-}\right)\right\} .
\end{aligned}
$$

We will show that $x_{3 \min } \neq\left(\rho_{1}, \rho_{2}, \theta_{1}^{+}, \theta_{2}^{+}\right)$. If $\sin \left(\theta_{1}^{+}-\theta_{2}^{+}\right)<0$, then $\rho_{1}=\rho_{1}^{+}$and $\rho_{2}=\rho_{2}^{-}$. Since $\rho_{1}^{+}>\rho_{2}^{-}$, we have $\frac{\partial \varphi}{\partial \theta_{1}}>0$, which violates the minimality condition for $\theta_{1}=\theta_{1}^{+}$. If $\sin \left(\theta_{1}^{+}-\theta_{2}^{+}\right)>0$, then $\rho_{1}=\rho_{1}^{-}$and $\rho_{2}=\rho_{2}^{+}$. Since $\rho_{1}^{-}<\rho_{2}^{+}$, we have $\frac{\partial \varphi}{\partial \theta_{2}}>0$, which violates the minimality condition with respect to $\theta_{2}=\theta_{2}^{+}$.

In the same way we can show that $x_{3 \max } \neq\left(\rho_{1}, \rho_{2}, \theta_{1}^{-}, \theta_{2}^{-}\right)$.

Before introducing algorithms for computing $\varphi^{-}$and $\varphi^{+}$we remind that if $\varphi^{+}<\varphi^{-}$, we just add $2 \pi$ to $\varphi^{+}$.

We summarize all the results of the discussion in the following two algorithms: 


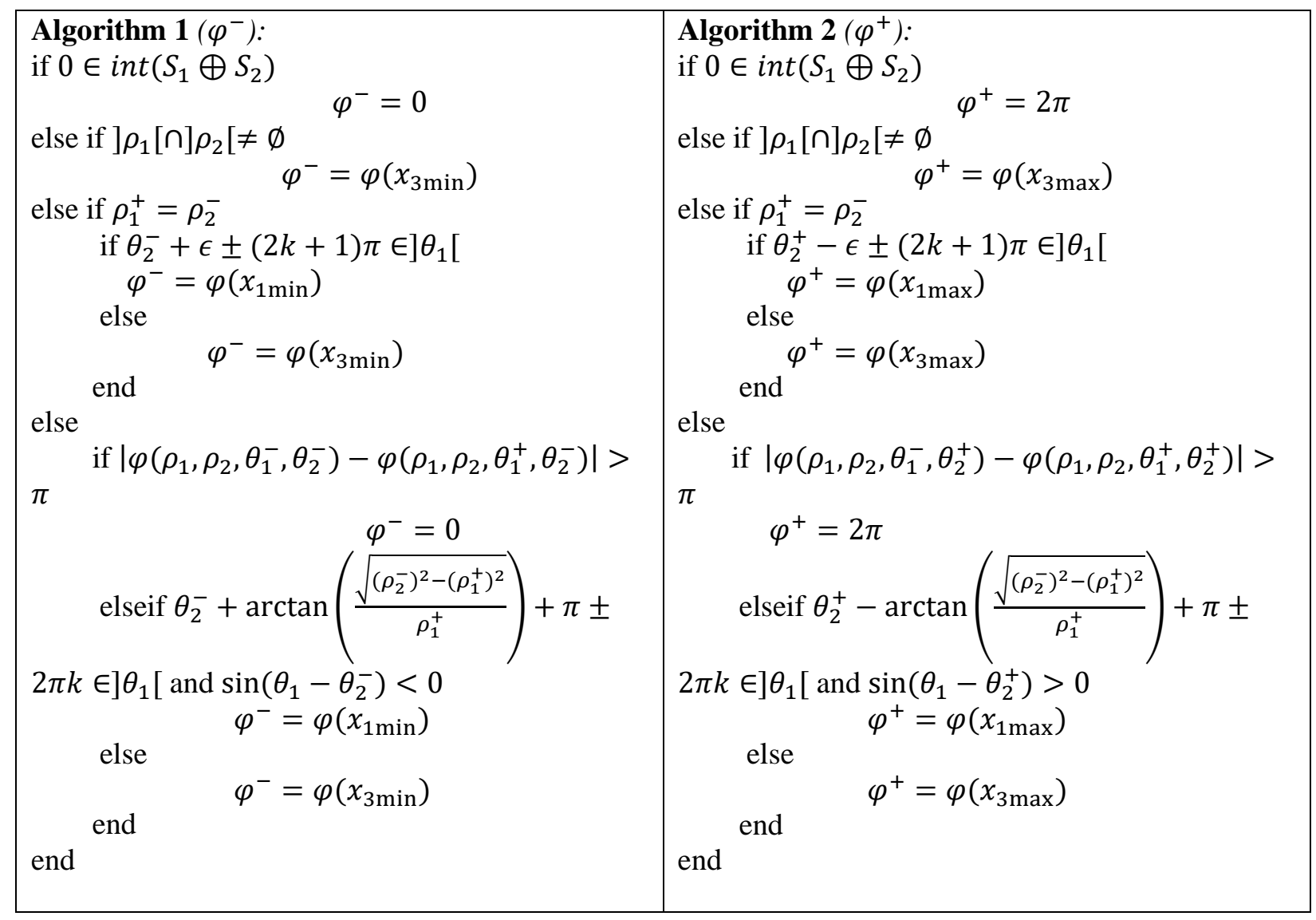

\subsection{Computing The Bounds of Magnitude Interval $[\rho]$}

Recall that,

$$
\rho=\sqrt{\rho_{1}^{2}+\rho_{2}^{2}+2 \rho_{1} \rho_{2} \cos (\theta)}
$$

where $\theta=\theta_{1}-\theta_{2} \in\left[\theta_{1}^{-}-\theta_{2}^{+}, \theta_{1}^{+}-\theta_{2}^{-}\right]$.

Since the square root function is an increasing function, we can minimize (maximize) $\rho$ by minimizing (maximizing) $h=\rho^{2}$, so the function that we are going to determine its extremes is,

$$
h: D \rightarrow(0, \infty) \text {, where } D=\left[\rho_{1}\right] \times\left[\rho_{2}\right] \times[\theta] \text {. }
$$

We aim to find two points $v_{\min }, v_{\max } \in D$ such that $\min h=h\left(v_{\min }\right), \max h=h\left(v_{\max }\right)$. The extremes of $h$ are located where $\nabla h=0$, that means,

$$
\frac{\partial h}{\partial \theta}=-2 \rho_{1} \rho_{2} \sin (\theta)=0, \frac{\partial h}{\partial \rho_{1}}=2 \rho_{1}+2 \rho_{2} \cos (\theta)=0, \frac{\partial h}{\partial \rho_{2}}=2 \rho_{2}+2 \rho_{1} \cos (\theta)=0 .
$$

The analysis after here is routine compared with the angle optimization. We ommit the proof and propose the following two improved algorithms for computing maxh and $\min h$ respectively. 


\begin{tabular}{|c|c|}
\hline $\begin{array}{l}\text { Algorithm } 3\left(\max \left(h=\rho^{2}\right)\right) \text { : } \\
\text { if } b \geq 0 \\
\qquad \max h=h\left(\rho_{1}^{+}, \rho_{2}^{+}, \theta\right) \\
\text { else } \\
=\operatorname{maxh} \\
\text { end }\end{array}$ & $\begin{array}{l}\text { Algorithm } 4\left(\min \left(h=\rho^{2}\right)\right) \text { : } \\
\text { if } a \geq 0 \quad \min h=h\left(\rho_{1}^{-}, \rho_{2}^{-}, \theta\right) \\
\left.\text { else if } \rho_{1}=\rho_{2}=0 \text { or }\left[\rho_{1}\right] \cap \rho_{2}\right] \neq \emptyset \text { and } \\
\pm(2 k+1) \pi \in\left[\theta_{1}^{-}-\theta_{2}^{+}, \theta_{1}^{+}-\theta_{2}^{-}\right] \\
\quad \min h=0 \quad \min h=h\left(\rho_{1}^{*}, \rho_{2}^{-}, \theta\right) \\
\left.\text { else if } \rho_{1}^{*}=-\rho_{2}^{-} a \in\right] \rho_{1}[ \\
\text { else } \quad \operatorname{minh} \\
=\min \left(h\left(\rho_{1}^{-}, \rho_{2}^{-}, \theta\right), h\left(\rho_{1}^{-}, \rho_{2}^{+}, \theta\right), h\left(\rho_{1}^{+}, \rho_{2}^{-}, \theta\right)\right) \\
\text { end }\end{array}$ \\
\hline
\end{tabular}

\section{NUMERICAL EXAMPLES}

In this section, we provide numerical examples to show the efficiency and robustness of the proposed algorithms. We also compare our algorithms with the existing algorithms in [6].

Example 1. Consider the sectors,

$S_{1}=[2,3] e^{\left[\frac{5 \pi}{9}, \frac{4 \pi}{3}\right]}, S_{2}=[3,5] e^{\left[0, \frac{5 \pi}{6}\right]}$

Using our algorithms we obtain, $[\rho]=[0,8],[\varphi]=[4.71674,9.68658]$ (Figure 7).

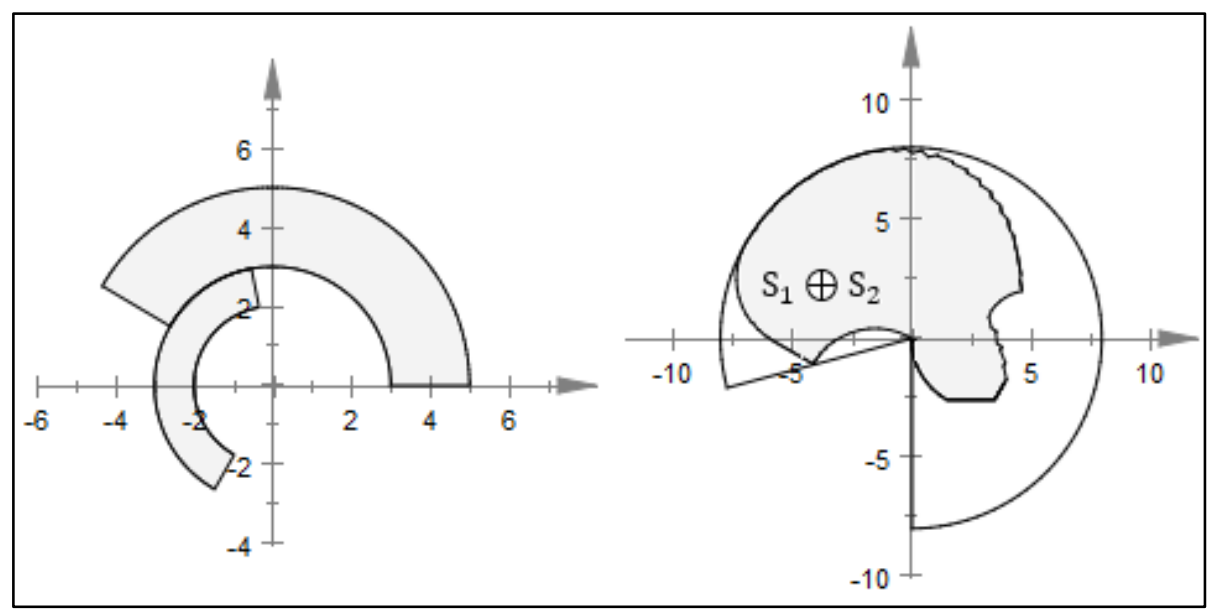

Figure 7. Graphs of Example 1

If we use the introduced algorithms in [7] we obtain $[\rho]=[0,8],[\varphi]=[0.4,3.4034]$, a result that is not correct.

Example 2. Consider the sectors,

$$
S_{1}=[2,3.5] e^{\left[\frac{5 \pi}{9}, \frac{11 \pi}{9}\right]}, S_{2}=[4,5] e^{\left[\frac{\pi}{18}, \frac{5 \pi}{6}\right]}
$$

Using our algorithms we obtain, $[\rho]=[0.5,8.5],[\varphi]=[5.39228,9.4654]$ (Figure 8). 


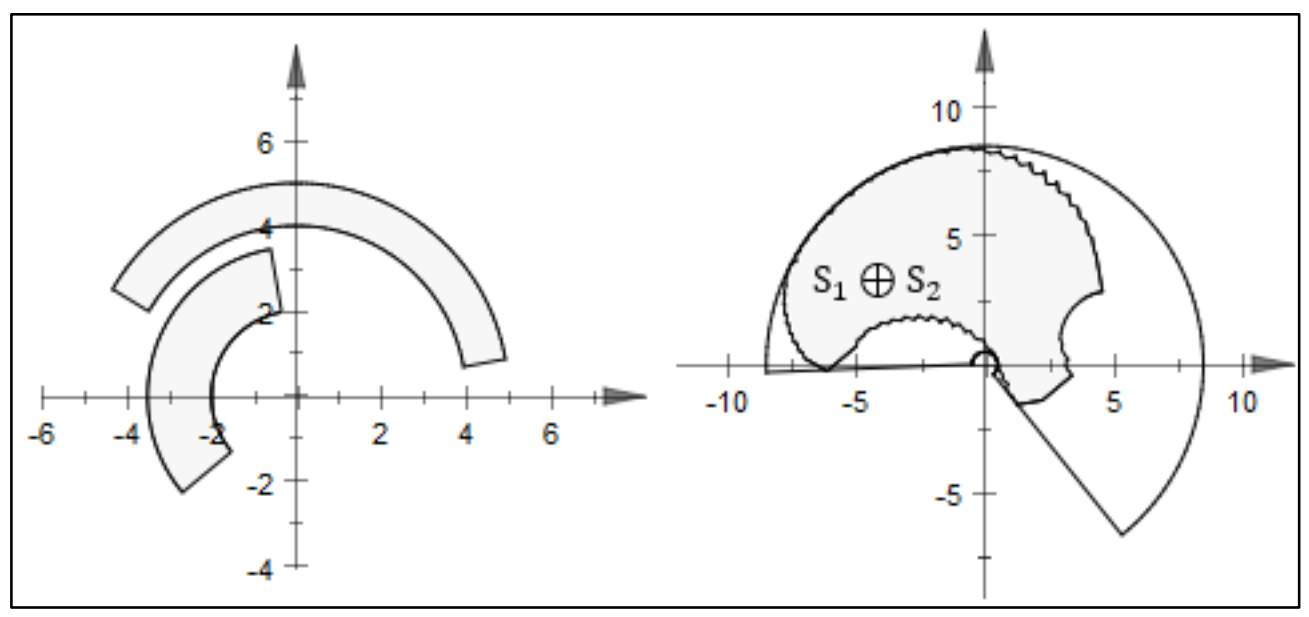

Figure 8. Graphs of Example 2

Using the proposed algorithms in $[7]$ we obtain $[\rho]=[0.5,8.5]$ and $[\varphi]=[0.555,3.1822]$, which is also not optimal.

\section{CONCLUSION}

From the results observed in the numerical examples, we conclude that the algorithms in [7] may fail to find the the optimal bounds for $\varphi$. As an alternative we developed simple and efficient algorithms to perform the addition (subtraction) operation of sectors. Numerical results demonstrate the efficiency, robustness and accuracy of the proposed algorithms. The algorithms are implemented in MATLAB R2020a environment and tested for all possible cases. For each case the result is computed for 12,960,000 sample points and compared with the outputs of the proposed algorithms. Figure 9 shows the user interface of the computer implementation.

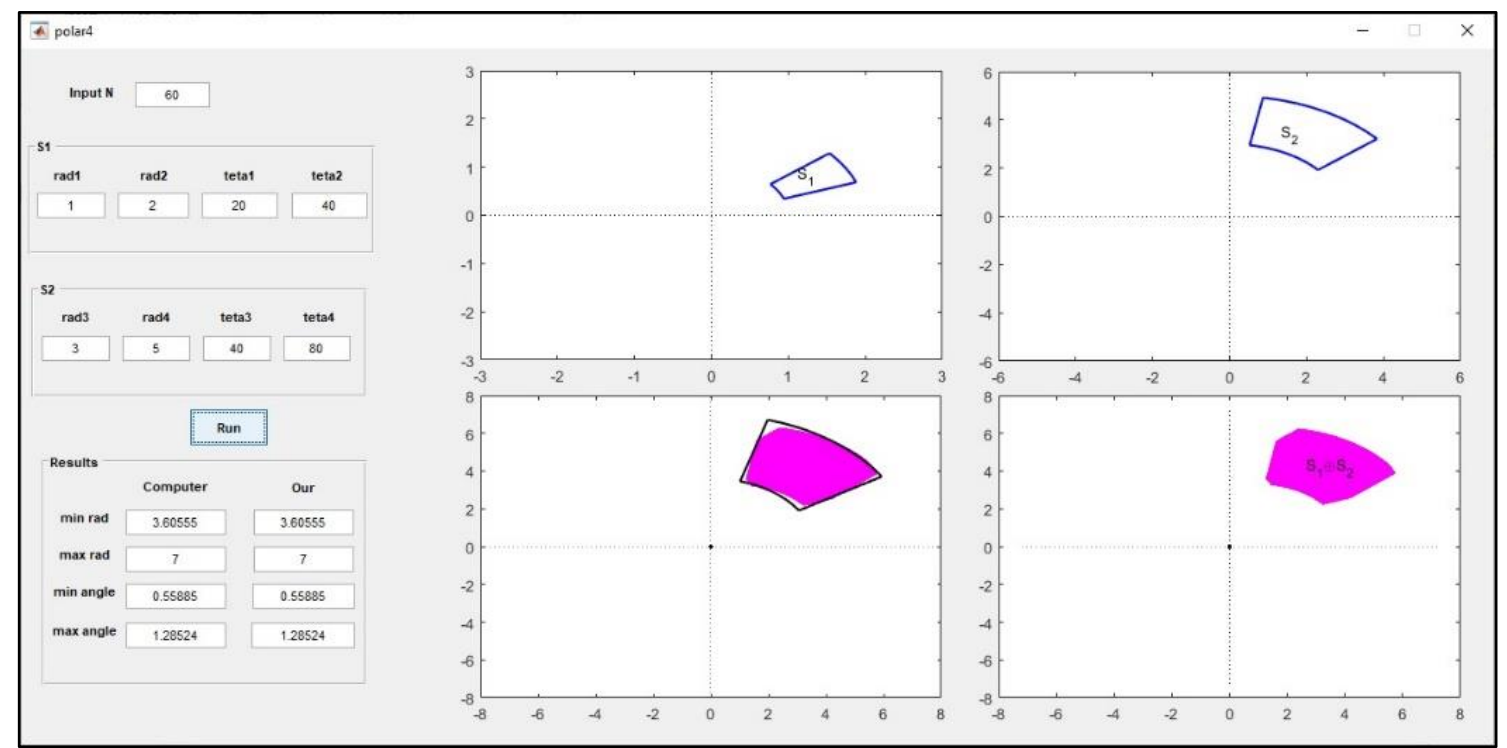

Figure 9. User Interface of the implemented Algorithms

\section{CONFLICTS OF INTEREST}

No conflict of interest was declared by the authors. 


\section{REFERENCES}

[1] Alefeld, G., Herzberger, J., Introduction to Interval Computations, Academic Press, New York, (1983).

[2] Mahmood, E. M.N., Soylu, G., "An effective method for division of rectangular intervals", AIMS Mathematics, 5(6): 6355-6372, (2020). DOI: 10.3934/math.2020409

[3] Boche, R., Complex Interval Arithmetic with Some Applications, Lockheed Missiles and Space Company, 4-22-66-1, Sunnyvale, California, (1966).

[4] Henri, P., "Circular arithmetic and the determination of polynomial zeros", Springer Lecture Notes, 228: 86-92, (1971).

[5] Klatte, R. and Ulrich, K., “Complex sector arithmetic”, Computing, 24(2): 139-148, (1980).

[6] Flores, J., "Complex Fans: A Representation for vectors in polar form with interval attributes", ACM Transactions on Mathematical Software, 25(2): 129-156, (1999).

[7] Candau, Y., Raissi, T., Ramdani, N., Ibos, L., "Complex interval arithmetic using polar form”, Reliable Computing, 12: 1-20, (2006). 